\title{
Awareness of Rabies Risk in a Sample of Travelers Attending an Irish Travel Medicine Clinic
}

\author{
Gerard Flaherty ${ }^{1,3,}$, John Gouda ${ }^{1}$, Graham Fry ${ }^{2}$ \\ 1. School of Medicine, National University of Ireland, Galway, Ireland \\ 2. Tropical Medical Bureau, Dublin, Ireland \\ 3. International Medical University, Kuala Lumpur, Malaysia
}

*Corresponding Author: Gerard Flaherty, School of Medicine, National University of Ireland, Galway, Ireland Email: gerard.flaherty@ nuigalway.ie

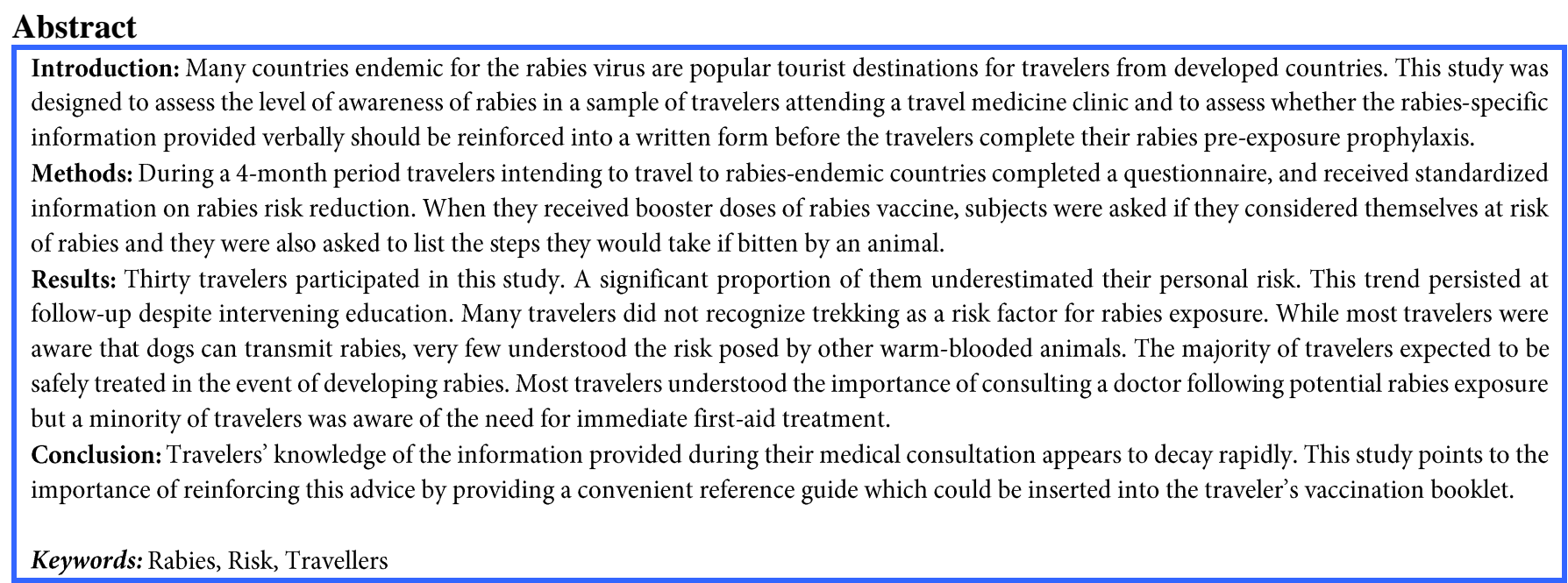

Article History: Received: 2 Mar 2015; Revised: 25 Apr 2015; Accepted: 1 May 2015

Cite this article as: Flaherty G, Gouda J, Fry G. Awareness of rabies risk in a sample of travelers attending an Irish travel medicine clinic. Int J Travel Med Glob Health. 2015;3(2):53-7.

\section{Introduction}

Rabies is a viral zoonosis caused by rhabdoviruses of the genus Lyssavirus. Infection of humans occurs when the rabies virus, carried in the saliva of a rabid animal, enters the body through penetrating bite wounds, open cuts in the skin, or contact with mucous membranes. Dogs are the most important reservoir of rabies infection worldwide, with more than $99.9 \%$ of human deaths from rabies resulting from dog bites [1]. Rabies causes an acute, progressive encephalomyelitis, which is almost always fatal [2]. There is no effective treatment to prevent deaths in humans exposed to the rabies virus once symptoms have appeared [3].

It is estimated that more than 50,000 deaths occur worldwide each year due to the rabies infection [4]. Most rabies infections occur in tropical and subtropical areas where the virus circulates in both domestic and stray animals [5]. Africa and Asia account for most of the cases due to rabies in humans worldwide, with the majority of cases being reported in India [3]. Many countries endemic for the rabies virus are popular tourist destinations for travellers from developed countries. Of 1,882 tourists who visited Thailand for an average of 17 days in one study, 24 received dog bites and $9 \%$ of the trav- ellers recalled that they were licked by dogs [6]. After receiving a bite from a rabid animal, pre-vaccinated patients are advised to thoroughly cleanse the wound with soap and water and to receive two booster doses of the vaccine on days 0 and 3 , in order to induce an anamnestic B cell response against the virus. Non-vaccinated patients must not only receive a full 28-day course of rabies vaccine on days $0,3,7$, and 14, but also a series of passive immunisations with human or equine rabies immunoglobulin [7]. For travellers to remote regions, where the risk of becoming exposed to rabies may be greater, it may be difficult or impossible to access safe post-exposure treatment without delay. The treatment may be prohibitively expensive for the uninsured backpacker on a tight budget and may be declined as a result [8]. Because of a shortage of rabies immunoglobulin in developing countries, less than $1 \%$ of those requiring the lifesaving treatment receive it [3].

This study was designed to assess the level of awareness of rabies in a sample of travellers attending an Irish travel medicine clinic. It was also designed to assess whether or not the information provided verbally during the initial consultation should be reinforced into a written form before the travellers complete their rabies pre-exposure prophylaxis. 


\section{Methods}

The ethics committee approval was not deemed necessary for this study. During a 4-month period, clients who expressed an intention to travel to rabies-endemic countries, were invited to participate in this study. Where a group of travelers attended the same consultation, one client was only invited to take part in the study. No information was provided on rabies until the 30 -item questionnaire was completed by the practice physician. Where open questions were used, the subject's own words were recorded verbatim. Subjects were advised to reply "don't know" where they did not have any knowledge of the answer to a particular item.

Following completion of the questionnaire, detailed information about rabies infection and its prevention was provided to the clients in a standardized manner. Where preexposure rabies prophylaxis was accepted by the study participant, the client was informed that a brief survey would be completed by the practice nurse following receipt of the third dose of rabies vaccine. The purpose of this follow-up questionnaire was to assess if patients correctly recalled the information on rabies they received during their initial consultation. Subjects were asked if they considered themselves to be at risk of rabies on their upcoming travels and if so, to what extent they were at risk.

Respondents were asked to list in sequence the practical steps they would take in the event of being exposed to rabies during their travels. The practice nurses recorded the client's own words without prompting. After the questionnaire was completed, the practice nurse gave specific preventive advice where knowledge of measures to be taken was lacking in individual travellers. Results were entered in a Microsoft Excel database and subsequently analysed by SPSS version 18.0 software.

\section{Results}

Thirty travellers were recruited into the study, 14 of whom were male and 16 were female. The average age of this cohort of travellers was 26.8 years (range: $19-54$ years). The majority of the participants were students $(30 \%, n=9)$. Only 2 travellers had previous medical training; one was a nurse and the other a third year medical student who had not yet started her Microbiology course.

The majority of the travellers (97\%) had at least 3 weeks remaining before their planned departure date. Although the duration of travel was not recorded in the questionnaire, each subject planned to spend at least 4 weeks in a rabiesendemic region. The majority of travellers $(77 \%, n=23)$ were travelling to Asia, with the majority of these visiting South East Asia, including Thailand, Cambodia and Vietnam. Twenty-three percent $(n=7)$ of the participants intended to visit South America, while only $7 \%(n=2)$ were traveling to Africa.

The travellers in this study intended to use a mixture of accommodation types, with $40 \%(n=12)$ staying exclusively in hotels. A large proportion of the sample $(57 \%, n=17)$ intended to trek during their holiday, with 2 travellers relying solely on tented accommodation throughout their trip. Reflecting the considerable degree of uncertainty about their itinerary in general, $40 \%(n=12)$ were unsure whether they would be trekking during their trip, but most were visiting areas where trekking is generally popular among tourists.

All 30 of the study subjects had heard of rabies before. Fifteen (50\%) erroneously believed that rabies was currently endemic in Ireland, while a further 5 individuals were unsure if rabies was present in Ireland. Ninety percent $(n=27)$ of those questioned reported that rabies is transmitted to humans via the saliva of an animal, but only 1 respondent mentioned that a scratch could be sufficient to transmit the disease. The majority of subjects $(83 \%, n=25)$ identified dogs as the principal animal responsible for spreading the disease to humans. Six subjects (20\%) implicated monkeys in the transmission of the disease, while 3 participants $(10 \%)$ believed that bats could infect humans with rabies. Two travellers were unable to name an animal carrier of the rabies virus. When asked how they would recognise rabies infection in an animal, 16 travellers (53\%) referred to "frothing at the mouth" or "foaming at the mouth", while 4 individuals (13\%) suggested that the animals may be more aggressive than usual. Nine travellers (30\%) either gave inappropriate responses to the question (e.g. "yellow eyes") or did not know how to recognize an animal with rabies. A single traveller correctly suggested that the rabid animal may appear perfectly normal.

Travellers were asked to identify activities or situations where they would anticipate an increased risk of being exposed to rabies. Ten (33\%) of those questioned identified trekking as a risk factor for rabies exposure. Many of the alternative responses were either incorrect or vague, and 27\% $(n=8)$ could not recall any situation which would confer an increased risk of rabies exposure. A third of the cohort $(n=10)$ admitted that they would pet dogs or cats in foreign countries. Eight respondents had previously received a bite from a dog, with 6 of these exposures occurring in Ireland and 1 each in the UK and Greece. Four travellers (13\%) did not consider themselves at risk of acquiring rabies during their next trip to a rabies-endemic country or were unsure of their level of risk (Figure 1).

For those travellers who acknowledged that they could become exposed to rabies, 8 (31\%) individuals declared themselves to be at low risk. Although the questionnaire did not specifically include the physician's assessment of the risk in individual cases, it is the authors' impression that every traveller was at moderate-to-high risk of rabies exposure due to the duration of travel and the proposed activities involved. Thirteen travellers (43\%) did not know how rabies would manifest itself clinically in an infected individual. Five travellers (17\%) believed that rabies was never fatal; of the 25 travellers who were aware that rabies may be fatal, the average fatality risk offered was $42 \%$. 
Twenty-one (70\%) participants incorrectly stated that an effective treatment exists for humans infected with the rabies virus. The majority of these travellers $(71 \%, \mathrm{n}=15)$ suggested that such treatment would be moderately to highly effective. The majority of subjects $(97 \%, n=29)$ were aware that a rabies vaccine existed which could prevent the disease.

When asked what measures they would take in the event of being exposed to rabies, 29 out of the 30 participants reported that they would seek urgent medical advice from a local doctor, clinic or hospital. Three subjects (10\%) declared that they would wash or disinfect the wound before seeking medical attention. All travellers stated that they would still seek medical advice even if the owner of the potentially rabid animal involved assured them that it was vaccinated against rabies. Five individuals (17\%) believed that further vaccination was unnecessary if they had received the appropriate course of pre-exposure vaccinations before their trip. A course of rabies vaccination was recommended in each traveller but was not possible in one case where only a week remained before departure and the subject was unwilling to receive the third booster dose at her destination. In the case of two subjects, the vaccine was declined due to needle phobia. A follow-up questionnaire was completed for 24 of the 27 travellers who had received the rabies vaccination regimen. All of these travellers deemed themselves to be at risk of being exposed to rabies at follow-up. Eight of these travellers (33\%) considered their risk of rabies exposure to be low (Figure 1). When subjects were asked what action they would take in the event of rabies exposure, all of them stated that they would seek a medical opinion without delay. Five travellers (21\%) specifically mentioned the necessity of receiving post-exposure vaccinations. Twelve subjects (50\%) stated that they would wash or disinfect the wound as a first-aid measure. Among the inappropriate responses received were the following: bleed the wound, consult family doctor in Ireland upon return, leave the country, capture the animal, and "suck out the poison".

\section{Discussion}

This is the first study to examine the issue of rabies awareness among travellers attending an Irish travel medicine clinic for pre-travel health advice. It is significant that such a large proportion (30\%) of the sample surveyed were students. Student travellers and backpackers on tight budgets are more likely to seek "only the essential shots" and may decline recommended vaccines such as rabies and hepatitis B for financial reasons.

It is noteworthy that, even though $77 \%$ of the travellers in this study planned on visiting Asia, which has the greatest reported incidence of human rabies infection, $13 \%$ of them still did not recognise that they were at risk and, of those that did, $31 \%$ believed that risks were low. With such a high percentage of the subjects trekking or potentially trekking (97\%) during their trip, it is reasonable to assume that their risk of being exposed to rabies was at least moderately high. Only a third of travellers in this study associated trekking with possible rabies exposure, highlighting the need for vigilance in this group. In a travel clinic in Kathmandu, Nepal, a common starting point for Himalayan treks, during a 3-year period, 56 travellers were treated for possible exposure to rabies, giving an annual incidence of 1.9 per 1,000 persons [9].

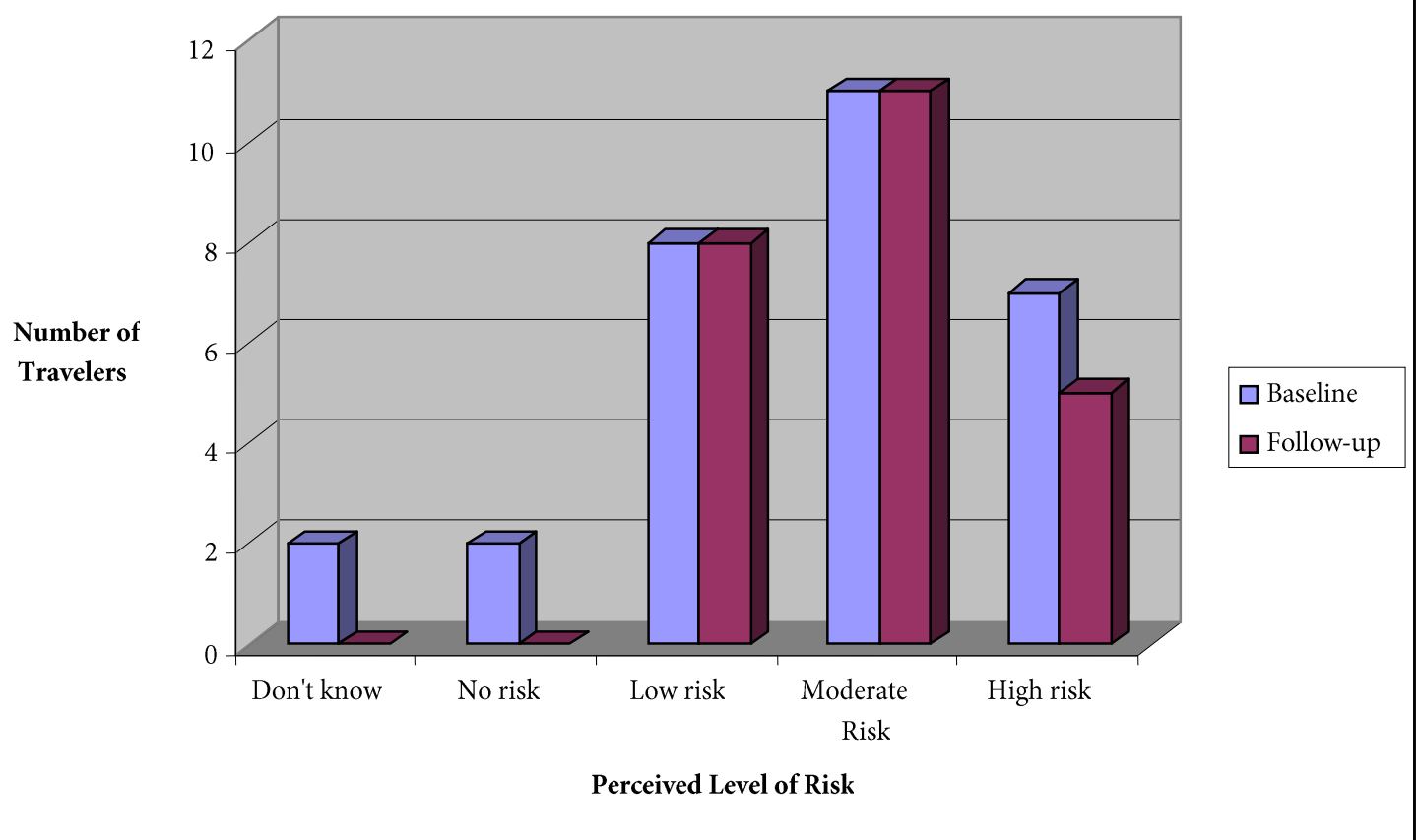

Figure 1. Travelers' perceived level of risk of rabies exposure 
While the majority of travellers were aware that dogs can transmit rabies to humans, a third of those surveyed admitted to petting dogs abroad and few travellers identified monkeys or bats as potential threats. This is a worrying observation, given that so-called "cryptic" human cases without a recognised exposure to a rabid animal, have become the norm in the United States of America. It is possible that a similar situation exists in South America, where vampire bat transmission of rabies is well recognized. Only 1 traveller stated that an animal scratch could result in rabies transmission, confirming our impression that this mode of transmission is not well recognized by the traveling public.

It is of concern that $17 \%$ of those questioned believed that rabies is not a fatal disease, with the case-fatality ratio being grossly underestimated at $42 \%$. Historically, 5 patients have survived rabies and in these cases some form of prophylaxis had been received [10]. Travellers who believe that a disease can be effectively treated may not appreciate the rationale behind vaccinating against that disease. In the present study, $70 \%$ of the travellers incorrectly assumed that rabies infection can be effectively treated and $17 \%$ believed that no further treatment was necessary if pre-exposure prophylaxis had been administered. Vaccinators should address this sense of complacency by ensuring that travellers clearly understand the role of pre-exposure prophylaxis and the need for post-exposure treatment following potential exposure [11].

While it is reassuring that most travellers in this study would seek urgent medical advice in the event of being exposed to rabies, only $10 \%$ of the study participants stated that they would take first-aid measures by washing the wound immediately. When questioned at follow-up 3-4 weeks after receiving their initial rabies vaccine, $33 \%$ of the travellers believed that they were at low risk of rabies exposure. It is likely that by underestimating their risk, these travellers may not exercise the caution required to avoid rabies exposure. It is interesting that the travellers did not revise their perceived level of risk in light of the pre-travel health advice they received.

It is encouraging that $21 \%$ of those surveyed at follow-up mentioned that they would require additional rabies vaccines following exposure. It is conceivable that some health professionals working in rural parts of developing countries may not have the necessary training to provide competent post-exposure advice. In a cohort of experienced travel health advisors in Germany, there were significant deficiencies highlighted in their assessment of specific rabies exposure scenarios [4]. In this situation, it is even more important for the vaccinated traveller to understand the necessity for post-exposure treatment.

When asked about the immediate steps they would take if exposed to rabies, $50 \%$ of those surveyed at follow-up mentioned that they would wash and/or disinfect the wound. This represents a significant improvement on the first-aid knowledge at baseline in these travellers but it does call into question the durability of the detailed advice given during the initial consultation. While the travel medicine clinic does provide an informative booklet reinforcing the pre-travel health advice given during the consultation, the section devoted to rabies is very brief and advises the travellers to seek immediate medical advice. This study underscores the need for a more detailed source of information, preferably one which the traveller can easily refer to in an emergency.

This observational study is limited by its small sample size which precluded a more detailed statistical analysis being performed. Because of the small numbers involved, no attempt was made to correlate the level of awareness of travellers at baseline and at follow-up with other variables, such as their demographic characteristics or travel itinerary.

\section{Conclusion}

Though their travel itinerary places them at considerable risk of rabies exposure, a significant proportion of travellers attending an Irish travel medicine clinic underestimate their personal risk. Travellers' knowledge of the information provided during their medical consultation decays rapidly. This study points to the importance of reinforcing this advice by providing a convenient reference guide to rabies, such as the example provided in Figure 2, which can be inserted into the traveller's vaccination booklet.

\section{Acknowledgements}

The authors wish to acknowledge the support of the administrative and clinical staff of the Tropical Medical Bureau clinic in Galway, Ireland.

\section{Authors' Contribution}

The authors were involved in the study design, data collection, interpretation of the results, and preparation of the manuscript.

\section{Funding/Support}

Not declared.

\section{Financial Disclosure}

None received. 
- Rabies is almost invariably fatal. No effective treatment exists.

- Most developing countries are high-risk areas for rabies exposure.

- A bite, scratch or lick from a warm-blooded animal, especially dogs, cats, monkeys and bats, may transmit the rabies virus.

- Bites on the head and neck are particularly dangerous.

- If you are in the company of young children, always suspect an animal exposure if the child is found crying and has been unsupervised in the presence of animals.

- Do not pet animals.

- Wear trousers if you are hiking or cycling. Be careful if you are jogging in big city slums.

- Avoid caves where bats often abound.

- If you have been pre-vaccinated and become exposed to rabies, wash the wound thoroughly with soap and water, apply an antiseptic and seek IMMEDIATE medical advice. It is preferable to visit a specialised centre where possible. The wound should NOT be stitched. Inform the doctor that you have been pre-vaccinated and require 2 further doses of cell-culture-derived rabies vaccine on days 0 and 3. You do NOT require rabies immunoglobulin in this situation. If this treatment is delayed it is never too late to receive it.

- If you have not been pre-vaccinated and become exposed to rabies, wash the wound thoroughly with soap and water, apply an antiseptic and seek IMMEDIATE medical advice. It is preferable to visit a specialised centre where possible. The wound should NOT be stitched. You require rabies immunoglobulin and FOUR doses of cell-culture-derived rabies vaccine on days $0,3,7$, and 14 . If this treatment is delayed it is never too late to receive it.

- Request a post-exposure treatment certificate from the doctor, detailing the type and quantity of vaccine used, the manufacturer's name, batch number, expiry date, route of administration and date of application.

- The vaccination status of the animal should not be a factor in withholding post-exposure treatment.

- Inform your travel medicine practitioner when you return from your trip.

Figure 2. Protect yourself against rabies

\section{References}

1. World Health Organization. World survey of rabies, No. 34. Geneva: WHO, 1998.

2. Field H, Macenzie J, Daszak P. Novel viral encephalitides associated with bats (Chiroptera) - host management strategies. Arch Virol Suppl. 2004;18:113-21.

3. Meslin FX. Rabies as a traveler's risk, especially in high-endemicity areas. J Travel Med. 2005;12:S30-S40.

4. Ross RD, Wolters B, Viazov SO, et al. Awareness of rabies risks and knowledge about preventive measures among experienced German travel health advisors. J Travel Med. 2006;13(5):261-67.

5. Warrell MJ, Warrell DA. Rabies and other lyssavirus diseases. Lancet. 2004;363(9413):959-69.

6. Phanuphak P, Ubolyam S, Sirivichayakul S. Should travellers in rabies endemic areas receive pre-exposure rabies immunisation? Ann Med Interne (Paris). 1994;145:409-11.

7. Ranney M, Partridge R, Jay GD. Rabies antibody seroprotection rates among travellers in Nepal: "Rabies seroprotection in travellers". J Travel Med. 2006;13(6):329-33.

8. Hoey J, Todkill A. Fatal case of rabies. Can Med Assoc J 1997;156:1311-12 (Editorial).

9. Pandey P, Shlim DR, Cave W, Springer MF. Risk of possible exposure to rabies among tourists and foreign residents in Nepal. J Travel Med. 2002;9:127-31.

10. Rupprecht CE, Gibbons RV. Prophylaxis against Rabies. N Engl J Med. 2004; 351:2626-35.

11. Toovey S, Moerman F, van Gompel A. Special infectious disease risks of expatriates and long-term travellers in tropical countries. Part II: Infections other than malaria. J Travel Med. 2007;14(1):50-60. 